\title{
Development of a Wireless Sensors Network for Greenhouse Monitoring and Control
}

\author{
Marwa Mekki ${ }^{*}$, Osman Abdallah ${ }^{2}$ \\ ${ }^{1}$ Faculty of Engineering and Technology, University of Gezira, Wad Medani, Sudan \\ ${ }^{2}$ Sudan Atomic Energy Commission, Sudan \\ e-mail: marwa_mekki@hotmail.com
}

\begin{abstract}
Wireless sensor networks (WSN) could be used to monitor and control many parameters of environment such as temperature, humidity, and radiation leakage. In greenhouse the weather and soil should be independent of the natural agents. To achieve this condition a wireless sensor nodes could be deployed and communicate with a central base station to measure and transmit the sensed required environment factors. In this paper a WSN was implemented by deployed wireless sensor nodes in a greenhouse with temperature, humidity, moisture light, and CO2 sensors. The proposed model was built and tested, and the result shows an excellent improvement in the sensed parameters. To control the environmental factors, the used microcontroller programmed to control the parameters according to preset values, or manually through a user interface panel.
\end{abstract}

Keywords: WSN, Wi-Fi, greenhouse, LabView

\section{Introduction}

A greenhouse (also called a glasshouse or a hothouse) is a building or complex in which plants are grown. These structures range in size from small sheds to industrial-sized buildings. A greenhouse is a structural building with different types of covering materials, such as a glass or plastic roof and frequently glass or plastic walls; it heats up because incoming visible sunshine is absorbed inside the structure. Air warmed by the heat from warmed interior surfaces is retained in the building by the roof and wall; the air that is warmed near the ground is prevented from rising indefinitely and flowing away. In domestic greenhouses the glass used is typically $3 \mathrm{~mm}$ (or $1 / 8 ")$ 'horticultural glass' grade, which is not generally as clear or free from imperfections as that used in a residential or office building [6].

Plastics mostly used are polyethylene film and multiwall sheets of . polycarbonate material, or PMMA acrylic glass [4].

Commercial glass greenhouses are often high-tech production facilities for vegetables or flowers. The glass greenhouses are filled with equipment such as screening installations, heating, cooling and lighting, and may be automatically controlled by a computer [5]. Climate Control Systems is sought after internationally for greenhouse automation technology [10].

During last decades, Wireless Sensor Networks (WSN) are used in many environmental monitoring applications, such as methane leak detection, Radioactive radiation leakage[1] Failure detection and industrial environment control [2], and in different applications such as Automated irrigation system [3]. This work proposed a wireless sensor network system for greenhouse monitoring and control. The network composed of sensor node with different sensors to measure and transmits the sensed data to a remote station via wireless communication. The control achieved by Arduino technology, and UGI is designed using LabView software in the gateway to show the received acquisition data [11-12]. The system is build with a single node but it can be extended to many others as much as needed, also the system measures some common parameters which can be increased as much as the node can carry.

\section{Materials and Method}

The system control and monitor some of the greenhouse parameters and make a decision for operation, and the hardware shown on the block diagram below. 


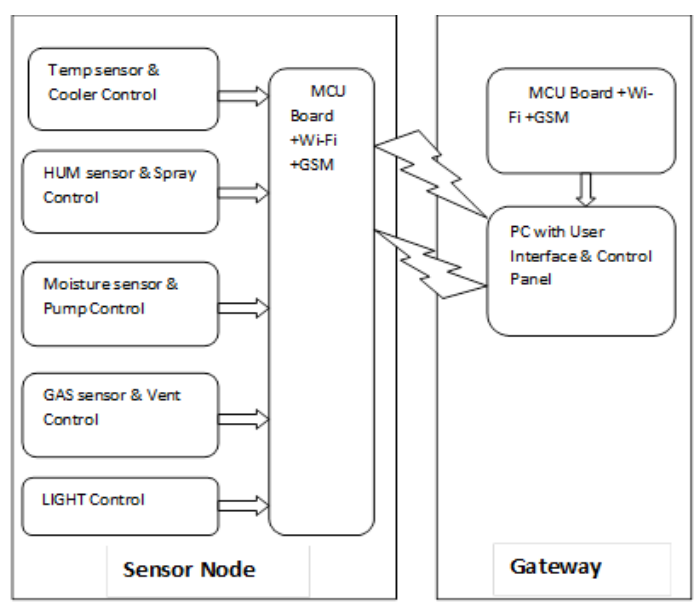

Figure 1. System Block Diagram

The system consists of a temperature sensor, humidity sensor, moisture sensor, light control and $\mathrm{CO} 2$ sensor. The temperature sensor monitors the temperature and if it goes above a preset value, the system turns on a cooler until the temperature get stable. The humidity is also monitored and a spray device is activated if humidity goes above limits. The sensor used to monitor temperature and humidity is $\mathrm{DHT} 11$ sensor which is a 1 wire interface temperature \& humidity sensor complex with a calibrated digital signal output.

The moisture is read using a moisture sensor SEN92355P from Grove technology, and if earth is dry, the system turns on a pump. A gas detector sensor FC-22-1 is used to detect $\mathrm{CO} 2$ percentage. If the percentage of $\mathrm{CO} 2$ is high, the system turns on a ventilator to purify the air. A light control system is attached in case of the need of longer lightening hours in addition to the normal lightening hours. All the preset parameters for temperature, humidity, moisture are user defined and depend on the plant grown and climate requirements.

\section{The Wireless System}

The proposed system composed of a sensor node to read the different greenhouse parameters and control a device with temperature, humidity, Gas and water sensors. The node communicates with a gateway via Freakduino board using Chibi Wi-Fi. The node also transmits the sensed data by GSM module to the gateway in case of remote or long distance monitoring.

\subsection{Communication}

Local communication network: Wi-Fi technology is implemented for the communication between sensor node and the gateway for short distance measurements in the wireless monitoring system network. This technology is a low cost and low power consumption technology. It provides $54 \mathrm{Mbps}$ data rate [5]. The current consumption is $350 \mathrm{~mA}$ that helps to prolong the battery life. The Wi-Fi technology used is Chibi Wi-Fi offered suitable number of nodes to cover the greenhouse with suitable distances between the node and the gateway (about 100m)

Global communication: A GSM technology is used to transmit the acquisitioned data for wide geographical area [7]. SIM900 is a complete Quad-band GSM/GPRS is used in the hardware. It is used for global communication. It supports voice uses $850 / 900 / 1800 / 1900 \mathrm{MHz}$ frequency ranges, SMS, Data, and Fax application in a small form factor and with low power consumption. For our application, SMS technique is used to send and receive data packages through AT commands [9].

\subsection{Control Unit}

An Arduino board compatible from FREAKLABS technology is used in both sensor node and gateway. The FreakLabs Freakduino is designed for rapid prototyping, 
experimentation, and deployment of wireless designs at low cost. It combines the ease-of-use of the Arduino IDE, compatibility with a rich assortment of third-party peripherals, and an integrated wireless radio for an inexpensive wireless prototyping system. The base board has all the functionality of an Arduino-based system that includes wireless communication and is an inexpensive way to start playing with wireless Arduino designs. It also has optional accessories such as a battery regulation circuit for battery-powered designs, bottom-mounted battery case to hold two AA batteries, and a ruggedized enclosure with integrated battery case. The addition of an integrated wireless radio based on the IEEE 802.15.4 protocol (same radio protocol as the $\mathrm{XBee}$ ) allows for wireless control of devices or wireless sensor data collection. Optional battery circuitry was added so that it could function as a true wireless node without any external power cables [8].

\section{Results}

Figure 2 shows the system hardware used and the connections established to control different parts in the sensor node. It consists of the main board, the sensors, GSM and Wi-Fi modules. Figure 3 shows the GUI designed and used to monitor and control the different components in the network. It consists of many fields described below. Each field monitor a parameter read from sensor node, and control a relative device as well.

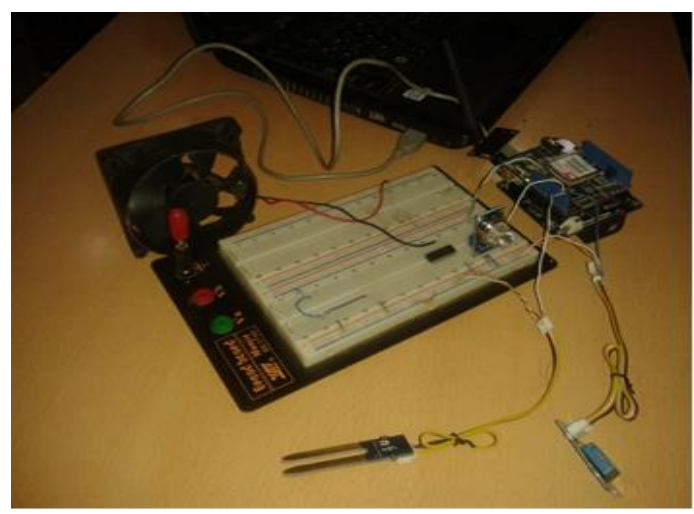

Figure 2. System hardware

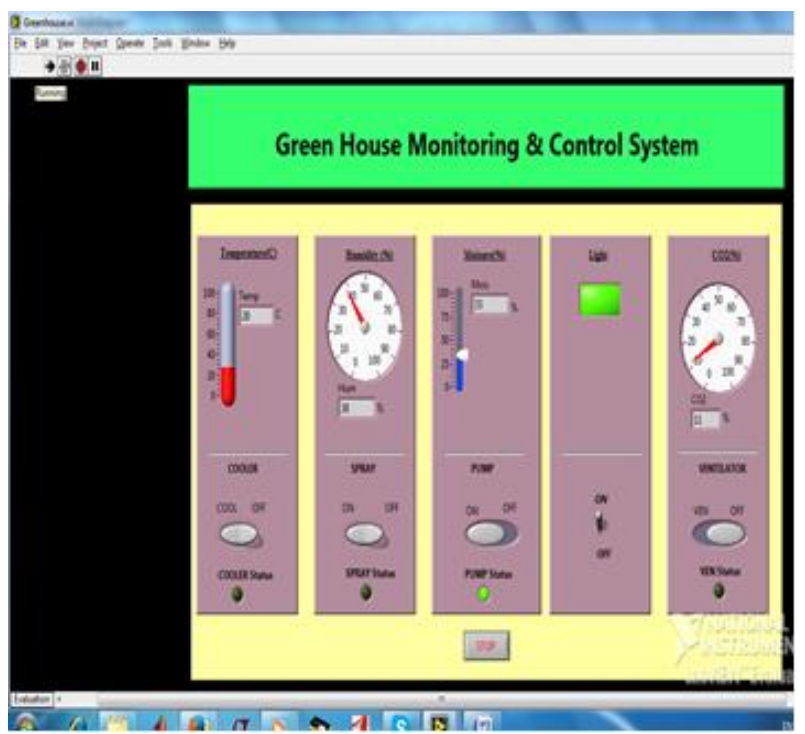

Figure 3. GUI for system monitor \& control

IJEEI Vol. 5, No. 3, September 2017 : $270-274$ 
Temperature field: displays the temperature and control a cooler. The cooler can be controlled manually from the control button on the front panel or automatically if the temperature exceeds a preset value.

Humidity field: monitors the percentage of the humidity and control a spray device. Humidity has a relation with the temperature, and also controlled manually or automatically.

Moisture field: shows the percentage of soil moisture and control a pump.

Light field: indicates the light status at the greenhouse and control a light bulb. ventilator.

CO2 field: monitor the percentage of harmful gases- especially $\mathrm{CO} 2$, and control a

Figure 4 shows the gateway system which consists of the Arduino board with the Wi-Fi and GSM modules, connected to the PC with the GUI in LabView.

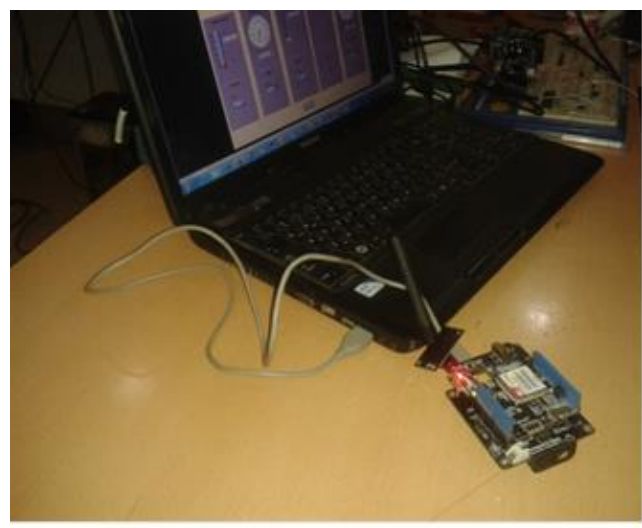

Figure 4. The gateway

Data package: The data sent from the sensor node to the gateway through the GSM modem come in form of an SMS package. The package contains parameter information of different sensors, which is extracted forward by the gateway software and displayed in the GUI panel. The same process is used to control the different devices in the sensor node from the gateway. The $\mathrm{Wi}-\mathrm{Fi}$ sends data between sensor node and gateway- and vice versa- in sequence to monitor and control the system. Figure 5 below shows the SMS frame which contains the information from the sensor node to the gateway. Each parameter read from the sensor node represented using a relative letter, for example: $\mathrm{T}$ for temperature, $\mathrm{H}$ : for humidity and so on. The value of the parameter is followed after the letter accordingly and each parameter separated from the other using a semicolon. When receiving the frame in the gateway software, each parameter read in specific bytes and displayed in the GUI.

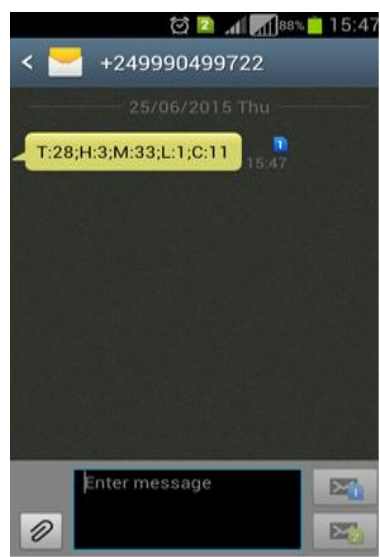

Figure 5. SMS data frame 


\section{Conclusion}

The purpose of this paper is to use the Wireless Sensor Network in greenhouse monitoring and control. The system is build to read different parameters in greenhouse like temperature, humidity, light, moisture and harmful gases. The system core is Arduino compatible technology and the WSN based on Wi-Fi is used for short distance communication and GSM for global system communication. A GUI panel is designed using LabView software to monitor and control the sensor node components and devices. All the parameters in the system could be adapted according to the plant type and climate requirements. The system is tested in the lab, and a field test could be performed for field verification.

\section{References}

[1] Andrey Somov, Alexander Baranov, Denis Spirjakin, Andrey Spirjakin, Vladimir Sleptsov, Roberto Passerone. Deployment and Evaluation of a Wireless Sensor Network for Methane Leak Detection. 2012.

[2] Ravishanker A, Pandian R. Embedded System Based Sensor Failure Detection and Industrial Environment Control over Wireless Network. 2014.

[3] Joaquin Gutlirrez, Juan Francisco Villa-Medina, Alejandra Nieto-Garibay, Maguel Parta-Gandara. Automated Irrigation System Using a Wireless Sensor Network and GPRS Module. IEEE. 2013.

[4] Roger Marshall. How to Build Your Own Greenhouse: Designs and Plans to Meet Your Growing Needs. ISBN 978-1-58017-587-6, (2006)

[5] Shame Smith, Marjorie Leggitt. Greenhouse Gardener's Companion, Revised: Growing Food \& Flowers in Your Greenhouse or Sunspace. Fulcrum Publishing, ISBN 1-55591-450-0, (2000)

[6] http://www.cambridgeglasshouse.co.uk/news/history-of-the-greenhouse

[7] http://www.seeedstudio.com/depot/gprs-shield-p-779.html)

[8] http://www.freaklabsstore.com/index.php?main_page=product_info

[9] Sim900_AT command_manual v1.03

[10] Vleeschouwer, Olivier de Greenhouses and conservatories. Flammarion, Paris, ISBN 2-08-010585-X.

[11] National Instrument, LabView, "Getting Started with LabView", Novemver2011 edition

[12] Boca Raton, "LabView Advanced programming Techniques", CRC press LLC. 2001. 